\title{
Trajectories of Postload Plasma Glucose in the Development of Type 2 Diabetes in Japanese Adults
}

\author{
Rie Oka, ${ }^{1,2}$ Kyoko Shibata, ${ }^{3}$ Masaru Sakurai, ${ }^{4}$ Mitsuhiro Kometani, ${ }^{2}$ Masakazu Yamagishi, ${ }^{2}$ \\ Kenichi Yoshimura, ${ }^{3}$ and Takashi Yoneda ${ }^{2}$ \\ ${ }^{1}$ Department of Internal Medicine, Hokuriku Central Hospital, Kanazawa University Hospital, Kanazawa, Japan \\ ${ }^{2}$ Department of Cardiovascular and Internal Medicine, Kanazawa University Graduate School of Medicine, Kanazawa, Japan \\ ${ }^{3}$ Department of Biostatistics, Innovative Clinical Research Center (iCREK), Kanazawa University Hospital, Kanazawa, Japan \\ ${ }^{4}$ Department of Social and Environmental Medicine, Kanazawa Medical University, Kanazawa, Japan \\ Correspondence should be addressed to Rie Oka; ririoka@goo.jp
}

Received 14 June 2017; Revised 15 August 2017; Accepted 22 August 2017; Published 14 September 2017

Academic Editor: Ed Randell

Copyright ( 2017 Rie Oka et al. This is an open access article distributed under the Creative Commons Attribution License, which permits unrestricted use, distribution, and reproduction in any medium, provided the original work is properly cited.

We aimed to clarify how the trajectories of 1-hour postload plasma glucose (PG) and 2-hour PG were different in the development of type 2 diabetes. Using data of repeated health checkups in Japanese workers from April 2006 to March 2016, longitudinal changes of fasting, 1-hour, and 2-hour PG on the oral glucose tolerance test were analyzed with a linear mixed effects model. Of the 1464 nondiabetic subjects at baseline, 112 subjects progressed to type 2 diabetes during the observation period (progressors). In progressors, 1-hour PG and 2-hour PG showed gradual increases with slopes of $1.33 \pm 0.2$ and $0.58 \pm 0.2 \mathrm{mg} / \mathrm{dL} / \mathrm{year}$, respectively, followed by a steep increase by which they attained diabetes. Until immediately before the diabetes transition, age- and sex-adjusted mean level of 2-hour PG was $149 \pm 2.7 \mathrm{mg} / \mathrm{dL}, 34 \pm 2.7$ (30\%) higher compared to nonprogressors, while that of 1-hour PG was $206 \pm 4.1 \mathrm{mg} / \mathrm{dL}, 60 \pm 4.3 \mathrm{mg} / \mathrm{dL}(41 \%)$ higher compared to nonprogressors. In conclusion, diabetes transition was preceded by a mild elevation of 2-hour PG for several years or more. The elevation in 1-hour PG was larger than that of 2-hour PG until immediately before the transition to diabetes.

\section{Introduction}

Postload hyperglycemia is one of the early manifestations of impaired glucose metabolism. Conventionally, investigators defined postload hyperglycemia by 2-hour plasma glucose (PG) on the oral glucose tolerance test (OGTT) and demonstrated that the intervention at the stage of impaired glucose tolerance (IGT) was effective to prevent or delay the incidence of type 2 diabetes [1-3]. However, it has been recently reported that 1-hour PG has a superior predictive ability to 2-hour PG in three Western cohort studies [4-6] and in two Asian cohort studies [7, 8]. Cross-sectional investigations have also revealed that elevated 1-hour PG rather than 2-hour PG showed a stronger correlation with decreased insulin secretion assessed by insulinogenic index $[4,5,7]$ or acute insulin response on the intravenous glucose tolerance test (IVGTT) [9]. The measurement of 1-hour PG cannot be ignored to detect the individuals with impairment of postload glucose metabolism.

In a few studies on trajectories of postload PG in the development of type 2 diabetes, 2 -hour PG showed a gradual linear increase for more than 10 years, followed by a rapid deterioration before the diagnosis [10-12]. They reported that 2-hour PG on average was regulated within the normal range $(<140 \mathrm{mg} / \mathrm{dL})$ until 2 years before the onset of diabetes $[10,12]$. They and any other investigators have not reported the trajectory of 1-hour PG. We hypothesized that 1-hour PG might increase earlier than 2-hour PG in those who later developed diabetes, which made its predictive ability better.

The aim of this study, therefore, was to depict trajectories of postload PG levels during the development of type 2 diabetes, with an interest in the difference of 1-hour PG from 2-hour PG. The optimal cutoff point of 1-hour PG in this population was also estimated. 


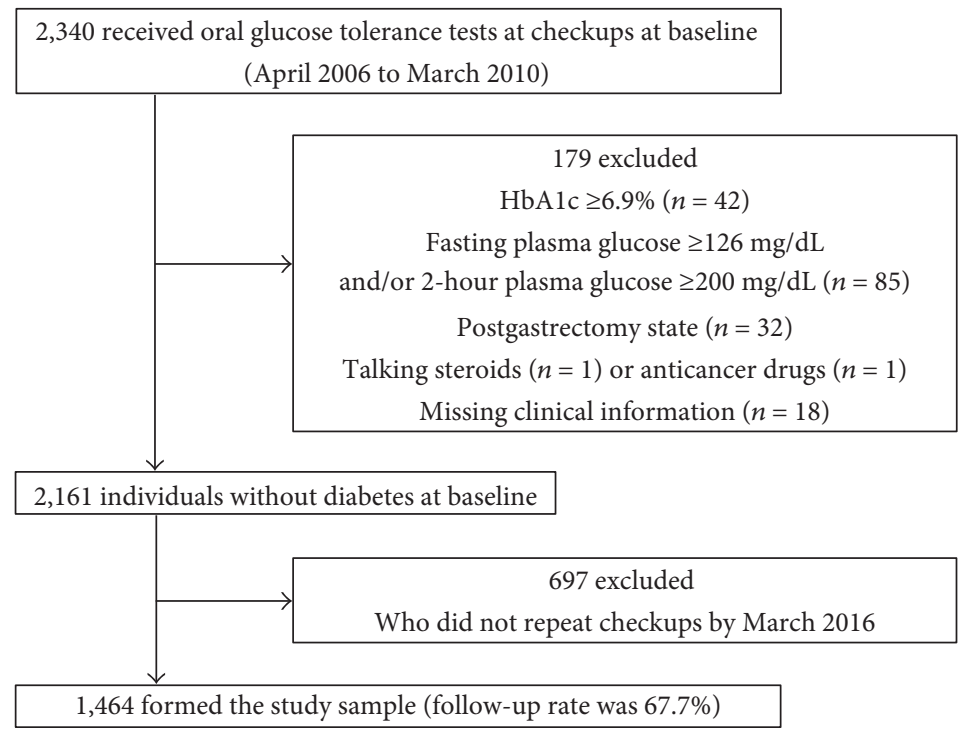

Figure 1: Study sample.

\section{Methods}

2.1. Study Subjects. A historical cohort study was conducted using data from the medical checkups of public school employees collected in the Hokuriku Central Hospital [7]. During April 2006 and March 2010 (baseline period), 2340 employees underwent an OGTT at a checkup [13]. If employees received more than one checkup during the baseline period, the initial checkup data were used. After those who had fasting $\mathrm{PG} \geq 126 \mathrm{mg} / \mathrm{dL}$ and/or 2-hour $\mathrm{PG} \geq 200 \mathrm{mg} / \mathrm{dL} \quad(n=85)$, who had HbAlc values $\geq 6.9 \%$ $(52 \mathrm{mmol} / \mathrm{mol})(n=42)$, who had undergone gastrectomy $(n=32)$, who were taking steroids $(n=1)$, who were taking anticancer drugs $(n=1)$, or who had any missing data $(n=18)$ were excluded, we selected 2161 nondiabetic individuals. Of the 2161 thus selected, 1464 individuals repeated checkups at least once by March 2016 and comprised our study sample (Figure 1). The subjects were followed until they developed diabetes; if they remained free of diabetes, follow-up ended at the time of the last checkup. The remaining 697 subjects did not repeat checkups, which meant a followup rate of $67.7 \%$. An OGTT was performed at all checkups during the follow-up period; but in the last two years (from April 2014 to March 2016), an OGTT was performed on 1191 subjects and the measurement of fasting PG only was performed in the remaining 273 subjects due to the financial reason of the mutual aid association. Information on smoking and drinking habits and medical history was obtained through a questionnaire. Subjects were considered current smokers if they smoked at least one cigarette per day. Alcohol use was defined by the number of days per week for drinking regardless of the amount consumed. Signed informed consent was obtained from all subjects, and the hospital review board approved the study protocol. The study was registered on the University Hospital Medical Information Network Clinical Trials Registry (UMIN-CTR, UMIN ID: UMIN000017662).
2.2. Diagnosis of Diabetes and Blood Sampling. Diabetes was diagnosed if fasting $P G \geq 126 \mathrm{mg} / \mathrm{dL}, 2$-hour $\mathrm{PG} \geq 200 \mathrm{mg} /$ $\mathrm{dL}$, and/or receiving treatments for type 2 diabetes based on the World Health Organization criteria (WHO) [14]. All the evaluations were performed at the health check department of the Hokuriku Central Hospital. Subjects were asked to visit the hospital between 8:00 a.m. and 9:00 a.m. after an overnight fast. At the baseline visit, an OGTT (75 g dextrose monohydrate in $250 \mathrm{ml}$ water) with $0,30,60$, and $120 \mathrm{~min}$ sampling to determine PG and insulin levels was performed on all subjects [13]. HbAlc assay was conducted at the central clinical laboratory in our hospital by a high-performance liquid chromatography (HPLC) method using ADAMS HA-8170 (ARKRAY, Kyoto). The following indices of insulin secretion and insulin sensitivity were calculated in this study: insulinogenic index $=\left(\operatorname{Ins}_{30}-\operatorname{Ins}_{0}[\mathrm{mU} / \mathrm{L}]\right) /\left(\mathrm{Gluc}_{30}-\mathrm{Gluc}_{0}[\mathrm{mg} / \mathrm{dL}]\right)$, where Ins $s_{\mathrm{y}}$ and $\mathrm{Glu}_{\mathrm{y}}$ represent values at time $y$ min during the OGTT [15] and homeostatic model assessment of insulin resistance $(\mathrm{HOMA})=\mathrm{Glu}_{0}(\mathrm{mg} / \mathrm{dL}) \times \operatorname{Ins}_{0}(\mathrm{mU} / \mathrm{L}) / 405[16]$.

2.3. Statistical Analysis. Analyses were conducted using SPSS software version 17.0 for Windows (SPSS Inc. Chicago, USA). Data are presented as the mean \pm SD or the median with the interquartile range for continuous variables or as a frequency for categorical variables. Subjects were divided into two groups, those who progressed to diabetes (progressors) and those who did not (nonprogressors). The differences in the baseline characteristics were tested using Student's $t$-test or Mann-Whitney $U$ tests for continuous variables or $\chi^{2}$ test for categorical values. The mixed effects model was used to estimate the trajectories of fasting PG, 1-hour PG, and 2hour PG [17]; this technique takes into account withinsubject correlations from repeated measurements with unequal numbers of observations per subjects and unequal intervals between measurements. As fixed effects, we entered time with sex and age as covariates into the model. We set a timeline with 0 year at the year of diagnosis for progressors or 
at the last checkup for nonprogressors, and time of prior measurements was coded backward, giving minus values. As a random effect, we included the participant's identification number. We used SPSS to calculate the annual estimated levels of PG and used $R$ to fit the model explaining the PG trajectories. The difference of the slopes of increase by time between progressors and nonprogressors was examined by the interaction between a dummy variable for progression $(0=$ nonprogressors; $1=$ progressors $)$ and time in the model. Finally, to identify the optimal cutoff point for progression of type 2 diabetes, subjects were divided by their baseline data at $140 \mathrm{mg} / \mathrm{dL}$ of 2 -hour PG based on the cutoff value for IGT [14], at $155 \mathrm{mg} / \mathrm{dL}$ of 1-hour PG based on the prior study by Abdul-Ghani et al. [18], and at $180 \mathrm{mg} / \mathrm{dL}$ of 1-hour PG based on the committee report of Japan Diabetes Society [19]. Then the hazard ratio (HR) of the development of diabetes of those over versus those below the cutoff point was calculated by Cox proportional hazard regression analysis. The HR was calculated using three models: Model 1, the multivariable-adjusted model, with covariates including age, sex, BMI, fasting PG, HbAlc, smoking status (three-level variable: current/former/never smoker), alcohol use (threelevel variable: drinking everyday/drinking 1-6 days per week/drinking less than 1 day per week), taking antihypertensive drugs (yes or no), and taking lipid-lowering drugs (yes or no); Model 2, in which Model 1 was additionally adjusted for the other PG values (i.e., 2-hour PG value for the division by 1-hour PG and 1-hour PG value for the division by 2-hour PG); and Model 3, in which Model 2 was further adjusted for insulinogenic index and HOMA index. A $p$ value $<0.05$ was considered statistically significant.

\section{Results}

3.1. Characteristics of Subjects. The study subjects were composed of 1464 subjects with a mean age of $52.0 \pm 7.0$ years and a mean BMI of $23.9 \pm 3.2 \mathrm{~kg} / \mathrm{m}^{2}$ at baseline. Of these, 112 subjects developed type 2 diabetes during the observation, diagnosed by fasting $\mathrm{PG} \geq 126 \mathrm{mg} / \mathrm{dL} \quad(n=51)$, 2-hour $\mathrm{PG} \geq 200 \mathrm{mg} / \mathrm{dL}(n=72)$, and/or receiving treatments for type 2 diabetes $(n=2)$. The remaining 1352 subjects stayed free of diabetes until follow-up. Table 1 presents the baseline characteristics of the two study groups: progressors and nonprogressors. Progressors included a higher proportion of men and had higher BMI, higher PG and insulin concentrations during OGTT, higher HbA1c, higher HOMA index, and lower insulinogenic index compared with nonprogressors $(p<0.05)$. Because 75 subjects had insulinogenic index values $\leq 0$, the analysis including insulinogenic index was performed on 1389 subjects.

3.2. Mixed Effects Model Analysis. A mixed effects model was used as this enables effective use of repeated measurements even when numbers and intervals of observations were different per subjects. The number of observations in each year was shown at the bottom of Figure 2. Fasting PG in nonprogressors showed a slight increase from $95 \pm 0.5 \mathrm{mg} /$ $\mathrm{dL}$ to $98 \pm 0.2 \mathrm{mg} / \mathrm{dL}$ during the 9 years of observation. In progressors, fasting PG showed a gradual increase until $>1$
TABLE 1: Baseline characteristics of the subjects.

\begin{tabular}{|c|c|c|}
\hline & Nonprogressors & Progressors \\
\hline$n$ & 1352 & 112 \\
\hline Age (years) & $52 \pm 7$ & $53 \pm 7$ \\
\hline Sex (\% male) & 69 & $79^{*}$ \\
\hline Body mass index $\left(\mathrm{kg} / \mathrm{m}^{2}\right)$ & $23.8 \pm 3.1$ & $25.2 \pm 3.7^{*}$ \\
\hline Fasting plasma glucose $(\mathrm{mg} / \mathrm{dL})$ & $95 \pm 7$ & $108 \pm 9^{*}$ \\
\hline 30 min plasma glucose $(\mathrm{mg} / \mathrm{dL})$ & $149 \pm 29$ & $185 \pm 29^{*}$ \\
\hline 1-hour plasma glucose $(\mathrm{mg} / \mathrm{dL})$ & $144 \pm 41$ & $209 \pm 32^{*}$ \\
\hline 2-hour plasma glucose $(\mathrm{mg} / \mathrm{dL})$ & $113 \pm 25$ & $148 \pm 31^{*}$ \\
\hline Fasting insulin (mU/L) & $2.8 / 3.8 / 5.1$ & 2.9/4.3/7.2* \\
\hline $30 \mathrm{~min}$ insulin $(\mathrm{mU} / \mathrm{L})$ & $19.2 / 28.6 / 45.2$ & $12.5 / 22.7 / 35.3^{*}$ \\
\hline HbAlc (\%) & $5.0 / 5.2 / 5.4$ & $5.4 / 5.6 / 5.9^{*}$ \\
\hline $\begin{array}{l}\text { Insulinogenic } \\
\text { index }(n=1389)\end{array}$ & $0.30 / 0.50 / 0.88$ & $0.14 / 0.24 / 0.43^{*}$ \\
\hline HOMA index & $0.66 / 0.90 / 1.26$ & $0.80 / 1.11 / 1.98^{*}$ \\
\hline Current smokers (\%) & 19.9 & $26.8^{*}$ \\
\hline Drinkers $(\%)$ & 64.3 & 59.8 \\
\hline $\begin{array}{l}\text { Antihypertensive } \\
\text { medications (\%) }\end{array}$ & 13.2 & $19.6^{*}$ \\
\hline $\begin{array}{l}\text { Lipid-lowering } \\
\text { medications (\%) }\end{array}$ & 6.8 & 8.0 \\
\hline
\end{tabular}

Data are expressed as mean $\pm S D, 25 / 50 / 75$ th percentile value, or number (\%). ${ }^{*} p<0.05$. HOMA: homeostasis model assessment of insulin resistance.

year, followed by a steep increase immediately before the diagnosis (from $112 \pm 2.6$ to $124 \pm 1.7 \mathrm{mg} / \mathrm{dL}$ ) (Figure 2(a)). A steep increase immediately before the diagnosis in progressors was also observed for 1-hour PG (from $220 \pm 5.4$ to $249 \pm 3.9 \mathrm{mg} / \mathrm{dL}$ ) (Figure 2(b)) and for 2-hour PG (from $155 \pm 5.4$ to $221 \pm 3.9 \mathrm{mg} / \mathrm{dL}$ ) (Figure 2(c)). Compared to $>9$ years, the estimated level was not significantly different in any other year but 0 year for 1-hour PG $(p<0.01)$ and for 2 -hour PG $(p<0.001)$. Age- and sex-adjusted mean level of 1 -hour PG until $>1$ year was $206 \pm 4.1 \mathrm{mg} / \mathrm{dL}, 60 \pm 4.3 \mathrm{mg} /$ dL $(41 \%)$ higher compared to nonprogressors $(p<0.001)$, and that of 2 -hour PG was $149 \pm 2.7 \mathrm{mg} / \mathrm{dL}, 34 \pm 2.7 \mathrm{mg} / \mathrm{dL}$ (30\%) higher compared to nonprogressors $(p<0.001)$. In 112 progressors, between the final and prefinal measurements of glucose concentrations, $42(38.5 \%)$ had a 1-year interval, $31(28.4 \%)$ had a 2-year interval, and the remaining $39(33.1 \%)$ had a 3 years or more interval, with a median value being 2 years in all progressors. When mixed effects model analysis was conducted in 28 progressors whose annual consecutive data were available until the diagnosis of diabetes, steep increases in 1-hour PG and 2-hour PG were also demonstrated (Supplementary Figure available online at https://doi.org/10.1155/2017/5307523).

To test this statistically, we fit a model including time as a continuous variable plus a dummy variable for 0 year $(1=0$ year; $0=$ from -9 to -1 year). The model including this dummy variable (piecewise model) fits better than the model with only continuous time variable (linear model) as assessed by Akaike information criterion (AIC). As shown in Table 2, 

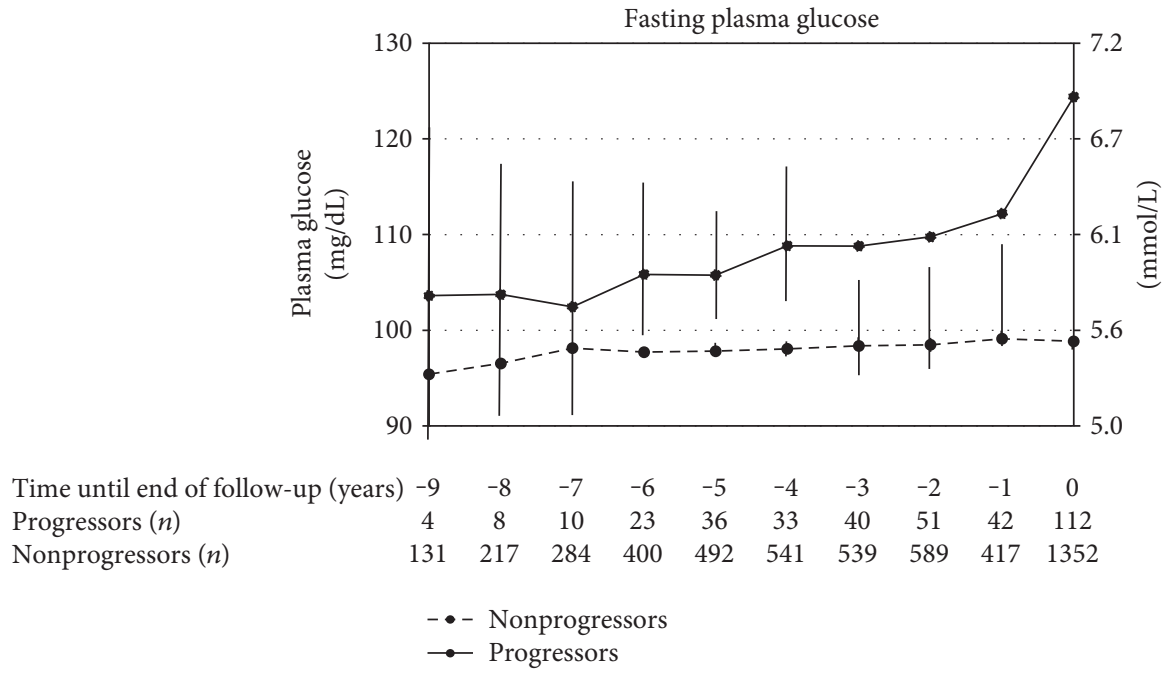

(a)

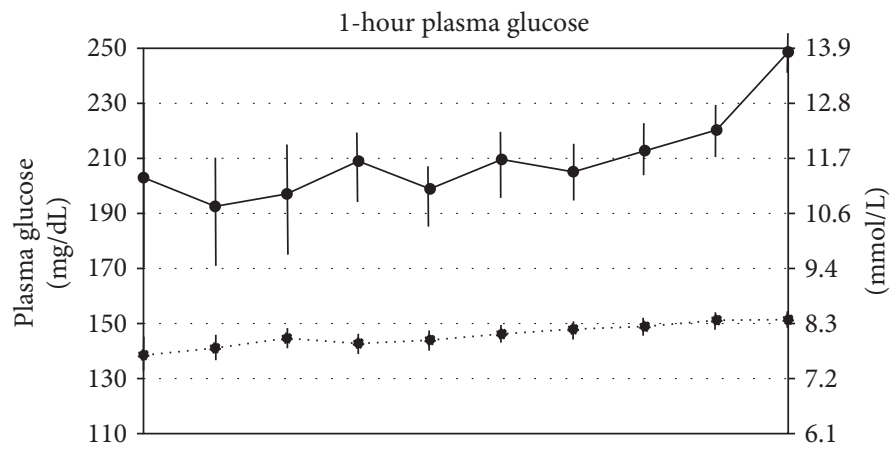

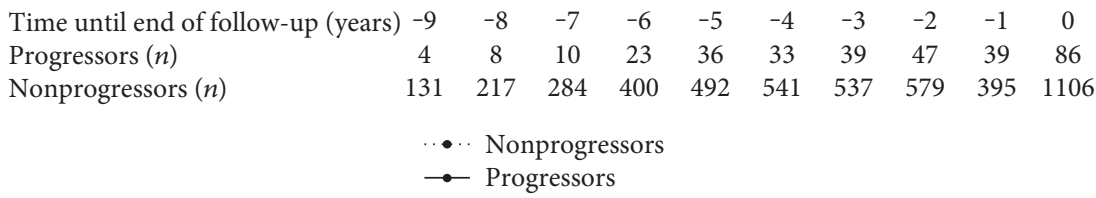

(b)

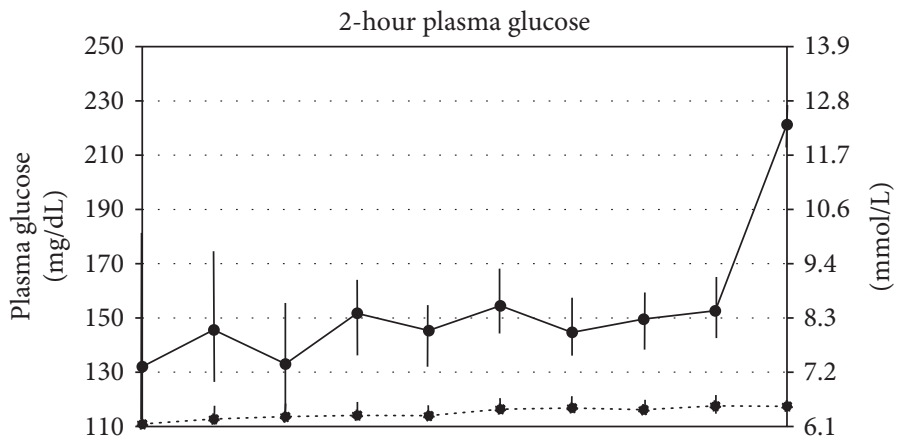

Time until end of follow-up (years) $-9 \begin{array}{llllllllll}-8 & -7 & -6 & -5 & -4 & -3 & -2 & -1 & 0\end{array}$

$\begin{array}{lllllllllll}\text { Progressors }(n) & 4 & 8 & 10 & 23 & 36 & 33 & 39 & 47 & 39 & 86\end{array}$

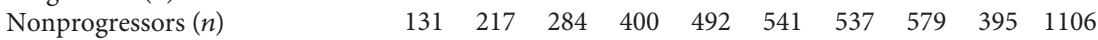

-.. Nonprogressors

$\rightarrow$ Progressors

(c)

Figure 2: Trajectories of fasting (a), 1-hour (b), and 2-hour (c) plasma glucose until the incidence of type 2 diabetes in 112 progressors compared to 1352 nonprogressors. Analyses were adjusted for age and sex in mixed effects models. Error bars show $95 \%$ confidence intervals for the estimated levels. 
TABLE 2: Fixed effects in the mixed effects models for changes of fasting, 1-hour, and 2-hour plasma glucose values in 112 progressors compared to 1352 nonprogressors.

\begin{tabular}{lccccc}
\hline & $\begin{array}{c}\text { Fasting PG }(\mathrm{mg} / \mathrm{dL}) \\
\text { Regression coefficient }\end{array}$ & SE & $\begin{array}{c}\text { 1-hour PG (mg/dL) } \\
\text { Regression coefficient }\end{array}$ & SE & $\begin{array}{c}\text { 2-hour PG (mg/dL) } \\
\text { Regression coefficient }\end{array}$ \\
\hline Intercept & 89.9 & 0.9 & 105.2 & 4.2 & 101.6 \\
Time (per year) & 0.22 & 0.0 & 1.33 & 0.2 & 0.58 \\
Progression & - & - & 49.9 & 7.9 & 2.7 \\
Progression $\times$ time & 1.23 & 0.2 & - & - & -2.0 \\
Progression $\times$ year 0 & 10.1 & 1.1 & 28.3 & 4.9 & - \\
\hline
\end{tabular}

Piecewise mixed effects modeling adjusted for age and sex. Time $=$ a continuous variable set at the year of diagnosis for progressors or at the last checkup for nonprogressors with 0 ; progression $=$ a dummy variable, 1 for progressors and 0 for nonprogressors; all coefficients are significant, $p<0.001$.

TABle 3: Hazard ratios (HR) for the development of diabetes by different cutoff points of 1-hour PG and 2-hour PG.

\begin{tabular}{|c|c|c|c|c|c|c|}
\hline \multirow{2}{*}{ Cutoff point } & \multirow{2}{*}{ Sensitivity (\%) } & \multirow{2}{*}{ Specificity (\%) } & \multirow{2}{*}{ Crude HR (95\% CI) } & \multicolumn{3}{|c|}{ Adjusted HR (95\% CI) } \\
\hline & & & & Model 1 & Model 2 & Model 3 \\
\hline $\begin{array}{l}\text { 1-hour PG } \geq 155 \mathrm{mg} / \mathrm{dL}(8.6 \mathrm{mmol} / \mathrm{L}) \\
\text { (yes versus no) }\end{array}$ & 92.9 & 60.3 & $18.2(8.9-37.4)$ & $7.7(3.6-16.3)$ & $5.9(2.7-12.7)$ & $5.1(2.2-11.8)$ \\
\hline $\begin{array}{l}\text { 1-hour } \mathrm{PG} \geq 180 \mathrm{mg} / \mathrm{dL}(10.0 \mathrm{mmol} / \mathrm{L}) \\
\text { (yes versus no) }\end{array}$ & 83.0 & 78.3 & $15.9(9.7-26.0)$ & $6.8(3.9-11.9)$ & $5.2(2.9-9.4)$ & $4.3(2.3-8.1)$ \\
\hline $\begin{array}{l}\text { 2-hour PG } \geq 140 \mathrm{mg} / \mathrm{dL}(7.8 \mathrm{mmol} / \mathrm{L} \text { ) } \\
\text { (yes versus no) }\end{array}$ & 60.7 & 83.4 & $7.3(5.0-10.8)$ & $3.1(2.0-4.8)$ & $1.6(1.0-2.6)$ & $1.6(1.0-2.6)$ \\
\hline
\end{tabular}

Model 1: adjusted for age, sex, body mass index, fasting PG, HbAlc, smoking status, alcohol use, taking antihypertensive drugs, and taking lipid-lowering drugs. Model 2: adjusted for Model 1 variables plus the other PG (i.e., 2-hour PG for cutoff of 1-hour PG and 1-hour PG for cutoff of 2-hour PG). Model 3: adjusted for Model 2 variables plus insulinogenic index and HOMA index.

large regression coefficients for progression (49.9 for 1-hour PG and 29.0 for 2-hour PG) indicated the difference between progressors and nonprogressors throughout the observation. The interaction term progression $\times 0$ year but not progression $\times$ time was significant both for 1 -hour and 2-hour PG, indicating that the slope of increase was not significantly different between progressors and nonprogressors until $>1$ year. When diabetes was defined only by fasting $\mathrm{PG} \geq 126 \mathrm{mg} / \mathrm{dL}$ and/or receiving treatments for type 2 diabetes; that is, individuals with isolated postload hyperglycemia were excluded from progressors, the results were similar (data not shown).

3.3. Predictive Abilities of Different Cutoff Points for 1-Hour $P G$ and 2-Hour PG. Table 3 shows the HR for the development of diabetes of those over versus those below the three different cutoff points. The HR of those with 1-hour $\mathrm{PG} \geq 155 \mathrm{mg} / \mathrm{dL}$ versus 1 -hour $\mathrm{PG}<155 \mathrm{mg} / \mathrm{dL}$ and the $\mathrm{HR}$ of those with 1 -hour $\mathrm{PG} \geq 180 \mathrm{mg} / \mathrm{dL}$ versus 1 -hour $\mathrm{PG}<180 \mathrm{mg} / \mathrm{dL}$ were significantly elevated even after adjusted for 2-hour PG, insulinogenic index, and HOMA index. The elevated HR of those with 2-hour PG $\geq 140 \mathrm{mg} /$ $\mathrm{dL}$ versus 2 -hour $\mathrm{PG}<140 \mathrm{mg} / \mathrm{dL}$ was significant $(p<0.05)$ but was attenuated after further adjustments for 1-hour PG.

\section{Discussion}

In this study, we depicted trajectories of postload plasma glucoses during the development of type 2 diabetes in Japanese workers. In agreement with prior studies [10-12], diabetes transition was preceded by a mild elevation of 2-hour PG for many years where the slopes of increase were not significantly different between progressors and nonprogressors. The difference between progressors and nonprogressors was relatively large in 1-hour PG rather than 2-hour PG until immediately before the diagnosis. These trajectories help to understand why 1-hour PG better predicts the future development of type 2 diabetes than 2-hour PG.

A rapid glucose rise in 2-hour PG immediately before the onset of diabetes (from -1 to 0 year) confirmed in this study was consistent with several cohort studies [10-12, 20, 21]. In the Pima longitudinal study which comprised 20 years before the development of diabetes, an exponential increase in the 2-hour glucose levels occurred during the final 4 years preceding the onset [10]. Ferrannini et al. also reported that the natural history of diabetes was marked by a steep increase in 2-hour plasma glucose in 3 years or shorter time frame, in which they discussed instability paradigm, in individuals who are destined to become diabetic; insulin sensitivity and insulin secretion are more or less altered, generating a critical state of instability [11]. In such an unstable condition, a relatively small further change would result in a large, rapid rise in glucose concentrations. In the Whitehall II Study, the magnitude of this rapid increase in 2-hour PG was larger than $70 \mathrm{mg} / \mathrm{dL}$ from $>2$ to 0 year [12], which was comparable to ours $(68 \mathrm{mg} / \mathrm{dL}$ from $>1$ to 0 year). As diabetes was attained by such a rapid rise in the short time frame, the difference of 2-hour PG between progressors and nonprogressors does not become large until just before the diagnosis. 
Mild elevation in 2-hour PG levels continued for several years or more until just before the aforementioned rapid rise. During this period, the slope in 2-hour PG as well as 1-hour PG was not significantly different between progressors and nonprogressors, in agreement with the results from the Whitehall II Study [12]. Therefore, contrary to our hypothesis, 1-hour PG did not increase earlier or faster than 2-hour PG, but the difference between progressors and nonprogressors present at the commencement of the observation was maintained for many years. As the difference in 1-hour PG was relatively larger rather than 2-hour PG until immediately before the diagnosis, it would be natural that progressors and nonprogressors were better discriminated by 1-hour PG than 2-hour PG.

Higher 1-hour PG than 2-hour PG is common in nondiabetic people as demonstrated by the studies on the shape of glucose curve during the OGTT [22-24]; about half of the study subjects had monophasic glucose curves, with a peak during 30-90 min followed by a decrease during 90$120 \mathrm{~min}$. The second popular type was biphasic, who had a nadir by $90 \mathrm{~min}$ and an increase again during 90-120 min. Compared to subjects with biphasic shape, monophasic subjects had a worse insulin sensitivity, lower insulin secretion, higher 1-hour PG [22-24], and higher risk for type 2 diabetes [25]. The elevation of 1-hour PG might reflect the characteristic of these monophasic type of subjects. Whether the elevation of 1 -hour PG is acquired or inherent is not known, but elevated 1-hour PG is likely to be present in the very early years in the natural course of the development of type 2 diabetes.

The cutoff point of $155 \mathrm{mg} / \mathrm{dL}$ for 1 -hour PG was first proposed by Abdul-Ghani et al. [18] and has been demonstrated to be useful to identify the individuals at higher risk for type 2 diabetes in other independent cohort studies from Finnish [5], Jewish [8], and Asian Indian populations [26]. Abdul-Ghani et al. set $155 \mathrm{mg} / \mathrm{dL}$ based on the result that the sum of sensitivity and specificity was maximal $(0.75$ and 0.79 for sensitivity and specificity, resp.), and they also reported that the optimal cutoff point for fasting PG was as low as $94 \mathrm{mg} / \mathrm{dL}$ applying the same method [18]. Indeed, the HR for future diabetes was significantly elevated both in NGT and IGT with 1 -hour PG $155 \leq \mathrm{mg} / \mathrm{dL}$ in this study. However, $44 \%$ of our study subjects had 1-hour PG $155 \leq \mathrm{mg} / \mathrm{dL}$ at baseline. Such a lower cutoff point might bring problems with socioeconomic costs as the new definition of "prediabetes" did so [27, 28]. As Asians have greater glycaemic excursions after taking glucose than Europeans [29], the ethnicity may also be taken into account to set the optimal cutoff point for 1-hour PG.

The strength of our study is the longitudinal observation of postload plasma glucoses, which allow for the comparison between the trajectory of 1-hour and that of 2-hour PG. However, several limitations of this study should be considered. First, data on subjects who did not receive checkups during the follow-up period were not available. Subjects who missed checkups might be less conscious about their health, which biased the study subjects toward metabolically healthy people. However, baseline characteristics including BMI, glucose, and insulin concentrations between the subjects who were followed and those who were missed were not significantly different. Second, 60 out of 112 progressors were diagnosed as diabetes only by 2 -hour PG $200 \leq \mathrm{mg} / \mathrm{dL}$ with normal fasting PG $(<126 \mathrm{mg} / \mathrm{dL})$, and the estimated value of fasting $\mathrm{PG}$ at the diagnosis (0 year) in progressors was relatively low compared to the prior studies. Our results may reflect more the character of isolated postload hyperglycemia rather than combined fasting and postload hyperglycemia. However, when diagnosis of diabetes was conducted only by fasting PG and/or receiving treatments, the results were similar. Third, the measurement of PG concentrations relied on a single OGTT, which is known to have within-subject variability [30].

In conclusion, longitudinally observed, diabetes transition was preceded by a mild elevation of 2-hour PG for many years. The difference in 1-hour PG between progressors and nonprogressors was relatively large until immediately before the diagnosis.

\section{Conflicts of Interest}

The authors declare that there are no conflicts of interest associated with this manuscript.

\section{Acknowledgments}

The authors thank the subjects and the staff at the Health Check Department of Hokuriku Central Hospital, Dr. Toru Aizawa at Aizawa Hospital for his thoughtful comments, and Dr. Kota Suzuki at Aichi Medical University for the advice on statistical analyses.

\section{References}

[1] W. C. Knowler, E. Barrett-Connor, S. E. Fowler et al., "Reduction in the incidence of type 2 diabetes with lifestyle intervention or metformin," The New England Journal of Medicine, vol. 346, pp. 393-403, 2002.

[2] J. Tuomilehto, J. Lindstrom, J. G. Eriksson et al., "Prevention of type 2 diabetes mellitus by changes in lifestyle among subjects with impaired glucose tolerance," The New England Journal of Medicine, vol. 344, pp. 1343-1350, 2001.

[3] E. M. Balk, A. Earley, G. Raman, E. A. Avendano, A. G. Pittas, and P. L. Remington, "Combined diet and physical activity promotion programs to prevent type 2 diabetes among persons at increased risk: a systematic review for the community preventive services task force," Annals of Internal Medicine, vol. 163, pp. 437-451, 2015.

[4] M. A. Abdul-Ghani, K. Williams, R. A. DeFronzo, and M. Stern, "What is the best predictor of future type 2 diabetes?," Diabetes Care, vol. 30, pp. 1544-1548, 2007.

[5] M. A. Abdul-Ghani, V. Lyssenko, T. Tuomi, R. A. DeFronzo, and L. Groop, "Fasting versus postload plasma glucose concentration and the risk for future type 2 diabetes: results from the Botnia Study," Diabetes Care, vol. 32, pp. 281-286, 2009.

[6] A. Alyass, P. Almgren, M. Akerlund et al., "Modelling of OGTT curve identifies $1 \mathrm{~h}$ plasma glucose level as a strong predictor of incident type 2 diabetes: results from two prospective cohorts," Diabetologia, vol. 58, pp. 87-97, 2015. 
[7] R. Oka, T. Aizawa, S. Miyamoto, T. Yoneda, and M. Yamagishi, "One-hour plasma glucose as a predictor of the development of type 2 diabetes in Japanese adults," Diabetic Medicine, vol. 33, pp. 1399-1405, 2016.

[8] M. Bergman, A. Chetrit, J. Roth, R. Jagannathan, M. Sevick, and R. Dankner, "One-hour post-load plasma glucose level during the OGTT predicts dysglycemia: observations from the 24year follow-up of the Israel Study of glucose intolerance, obesity and hypertension," Diabetes Research and Clinical Practice, vol. 120, pp. 221-228, 2016.

[9] M. A. Marini, E. Succurro, S. Frontoni et al., "Insulin sensitivity, $\beta$-cell function, and incretin effect in individuals with elevated 1-hour postload plasma glucose levels," Diabetes Care, vol. 35, pp. 868-872, 2012.

[10] C. C. Mason, R. L. Hanson, and W. C. Knowler, "Progression to type 2 diabetes characterized by moderate then rapid glucose increases," Diabetes, vol. 56, pp. 2054-2061, 2007.

[11] E. Ferrannini, M. Nannipieri, K. Williams, C. Gonzales, S. M. Haffner, and M. P. Stern, "Mode of onset of type 2 diabetes from normal or impaired glucose tolerance," Diabetes, vol. 53, pp. 160-165, 2004.

[12] A. G. Tabak, M. Jokela, T. N. Akbaraly, E. J. Brunner, M. Kivimaki, and D. R. Witte, "Trajectories of glycaemia, insulin sensitivity, and insulin secretion before diagnosis of type 2 diabetes: an analysis from the Whitehall II study," Lancet, vol. 373, pp. 2215-2221, 2009.

[13] R. Oka, K. Yagi, M. Sakurai et al., "Insulin secretion and insulin sensitivity on the oral glucose tolerance test (OGTT) in middleaged Japanese," Endocrine Journal, vol. 59, pp. 55-64, 2012.

[14] World Health Organization ID, Definition and Diagnosis of Diabetes Mellitus and Intermediate Hyperglycaemia: Report of a WHO/IDF Consultation, World Health Organization, Geneva, 2006.

[15] K. Kosaka, T. Kuzuya, R. Hagura, and H. Yoshinaga, "Insulin response to oral glucose load is consistently decreased in established non-insulin-dependent diabetes mellitus: the usefulness of decreased early insulin response as a predictor of noninsulin-dependent diabetes mellitus," Diabetic Medicine, vol. 13, no. 9, Supplement 6, pp. S109-S119, 1996.

[16] D. R. Matthews, J. P. Hosker, A. S. Rudenski, B. A. Naylor, D. F. Treacher, and R. C. Turner, "Homeostasis model assessment: insulin resistance and beta-cell function from fasting plasma glucose and insulin concentrations in man," Diabetologia, vol. 28, pp. 412-419, 1985.

[17] G. M. L. N. Fitzmaurice and J. H. Ware, Applied Longitudinal Analysis, John Wiley \& Sons, New York, 2011.

[18] M. A. Abdul-Ghani, T. Abdul-Ghani, N. Ali, and R. A. DeFronzo, "One-hour plasma glucose concentration and the metabolic syndrome identify subjects at high risk for future type 2 diabetes," Diabetes Care, vol. 31, no. 8, pp. 1650-1655, 2008.

[19] Committee of the Japan Diabetes Society on the Diagnostic Criteria of Diabetes Mellitus, Y. Seino, K. Nanjo et al., "Report of the committee on the classification and diagnostic criteria of diabetes mellitus," Journal of Diabetes Investigation, vol. 1, no. 5, pp. 212-228, 2010.

[20] A. H. Xiang, C. Wang, R. K. Peters, E. Trigo, S. L. Kjos, and T. A. Buchanan, "Coordinate changes in plasma glucose and pancreatic beta-cell function in Latino women at high risk for type 2 diabetes," Diabetes, vol. 55, pp. 10741079, 2006.
[21] E. Laspa, A. Christen, Z. Efstathiadou, D. G. Johnston, and I. F. Godsland, "Long-term changes and variability in diabetes risk factors prior to the development of impaired glucose homeostasis," Diabetic Medicine, vol. 24, no. 11, pp. 12691278, 2007.

[22] M. Kanauchi, K. Kimura, K. Kanauchi, and Y. Saito, "Beta-cell function and insulin sensitivity contribute to the shape of plasma glucose curve during an oral glucose tolerance test in non-diabetic individuals," International Journal of Clinical Practice, vol. 59, pp. 427-432, 2005.

[23] O. Tschritter, A. Fritsche, F. Shirkavand, F. Machicao, H. Häring, and M. Stumvoll, "Assessing the shape of the glucose curve during an oral glucose tolerance test," Diabetes Care, vol. 26, no. 4, pp. 1026-1033, 2003.

[24] A. Tura, U. Morbiducci, S. Sbrignadello, Y. Winhofer, G. Pacini, and A. Kautzky-Willer, "Shape of glucose, insulin, Cpeptide curves during a 3-h oral glucose tolerance test: any relationship with the degree of glucose tolerance?," American Journal of Physiology. Regulatory, Integrative and Comparative Physiology, vol. 300, pp. R941-R948, 2011.

[25] M. A. Abdul-Ghani, V. Lyssenko, T. Tuomi, R. A. Defronzo, and L. Groop, "The shape of plasma glucose concentration curve during OGTT predicts future risk of type 2 diabetes," Diabetes/Metabolism Research and Reviews, vol. 26, pp. 280 286, 2010.

[26] M. Priya, R. M. Anjana, F. S. Chiwanga, K. Gokulakrishnan, M. Deepa, and V. Mohan, "1-hour venous plasma glucose and incident prediabetes and diabetes in Asian Indians," Diabetes Technology \& Therapeutics, vol. 15, pp. 497-502, 2013.

[27] T. M. Dall, W. Yang, P. Halder et al., "The economic burden of elevated blood glucose levels in 2012: diagnosed and undiagnosed diabetes, gestational diabetes mellitus, and prediabetes," Diabetes Care, vol. 37, pp. 3172-3179, 2014.

[28] J. S. Yudkin and V. M. Montori, "The epidemic of pre-diabetes: the medicine and the politics," BMJ, vol. 349, article g4485, 2014.

[29] M. Kataoka, B. J. Venn, S. M. Williams, L. A. Te Morenga, I. M. Heemels, and J. I. Mann, "Glycaemic responses to glucose and rice in people of Chinese and European ethnicity," Diabetic Medicine, vol. 30, pp. e101-e107, 2013.

[30] K. M. Utzschneider, R. L. Prigeon, J. Tong et al., "Within-subject variability of measures of beta cell function derived from a 2 h OGTT: implications for research studies," Diabetologia, vol. 50, no. 12, pp. 2516-2525, 2007. 


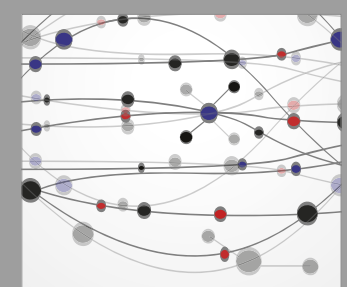

The Scientific World Journal
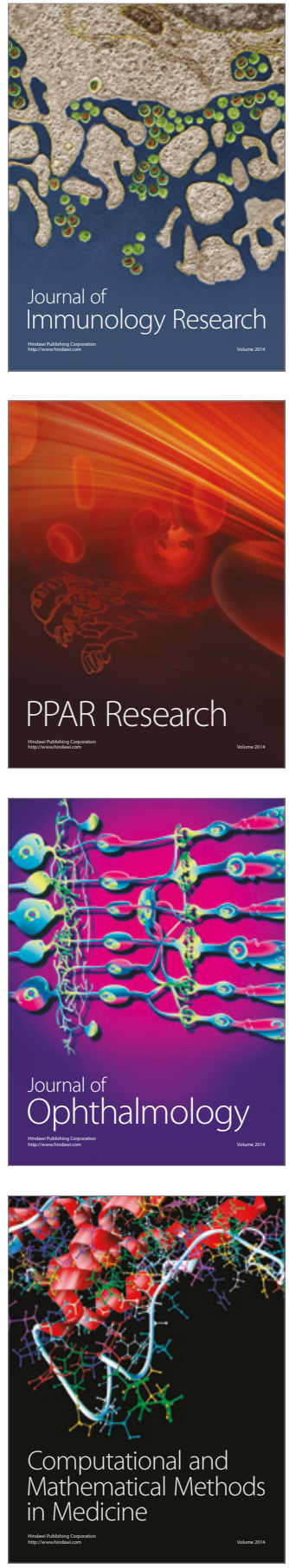

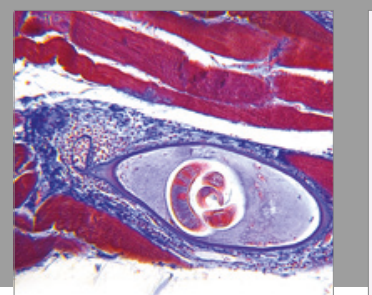

Gastroenterology Research and Practice
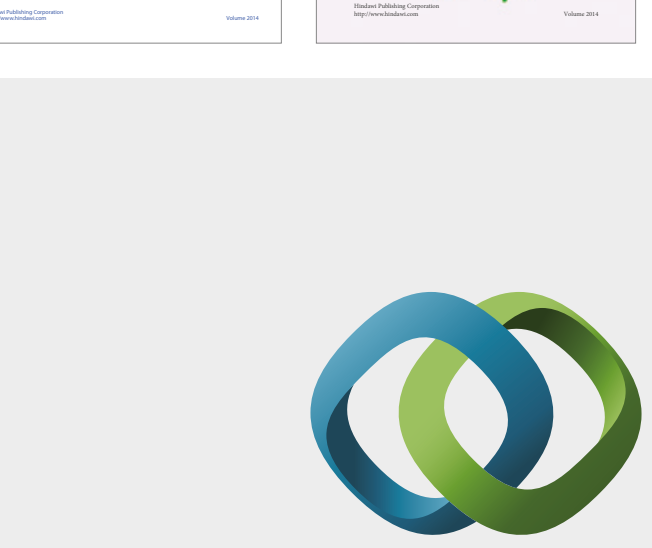

\section{Hindawi}

Submit your manuscripts at

https://www.hindawi.com
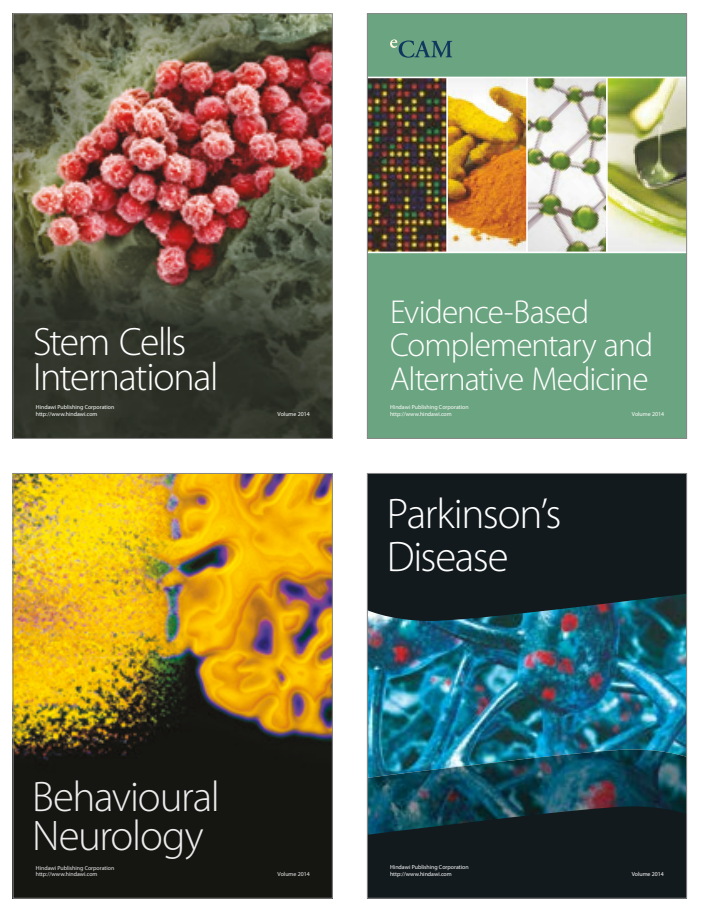
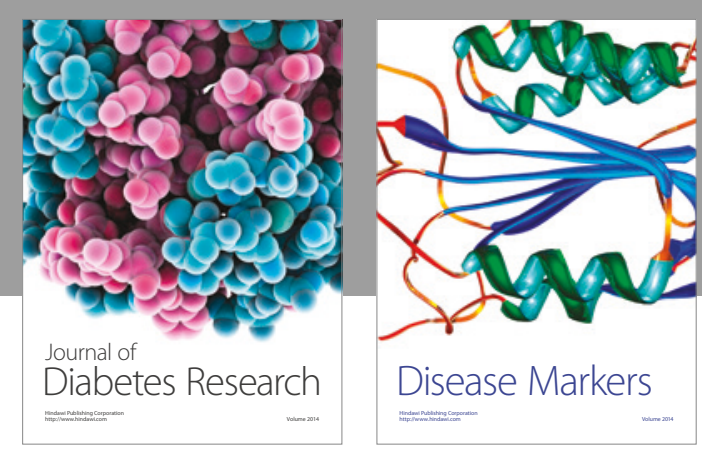

Disease Markers
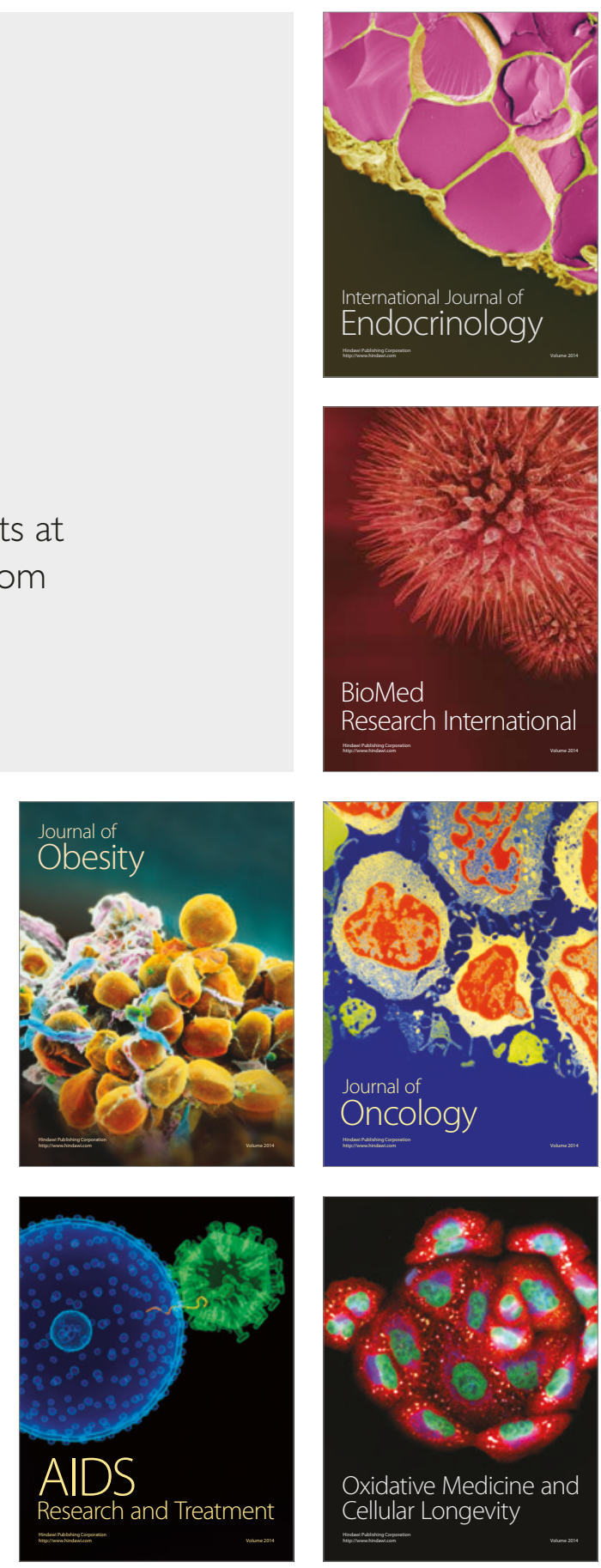